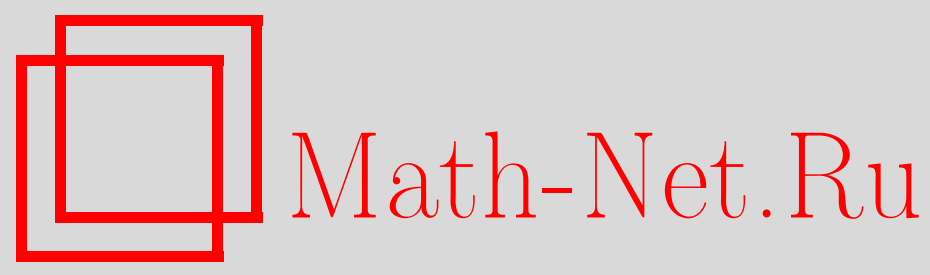

П. А. Крутицкий, Смешанная задача для уравнения Лапласа во внешней области с произвольным разбиением границы, Матем. заметки, 2001, том 69, выпуск 6, 876-891

DOI: https://doi.org/10.4213/mzm701

Использование Общероссийского математического портала Math-Net.Ru подразумевает, что вы прочитали и согласны с пользовательским соглашением http://www.mathnet.ru/rus/agreement

Параметры загрузки:

IP: 3.85 .5 .30

26 апреля 2023 г., 18:34:19

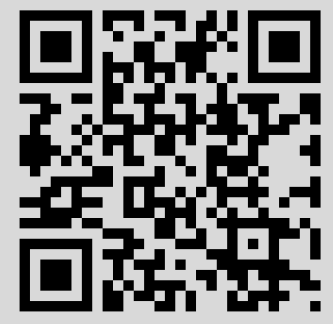




\title{
СМЕШАННАЯ ЗАДАЧА ДЛЯ УРАВНЕНИЯ ЛАПЛАСА ВО ВНЕШНЕЙ ОБЛАСТИ \\ С ПРОИЗВОЛЬНЫМ РАЗБИЕНИЕМ ГРАНИЦЫ
}

\section{П. А. Крутицкий}

\begin{abstract}
В работе предложен метод решения смешанной краевой задачи для уравнения Лапласа в связной внешней области с произвольньм разбиением границы. Все простые замкнутые кривые, составляющие границу, разбиты на три совокупности. На элементах первой из них задано условие Дирихле, на элементах второй - третье краевое условие, а третья, в свою очередь, разбита на две совокупности простых разомкнутых дуг, на элементах одной из которых задано условие Дирихле, а на элементах другой - третье краевое условие. Задача сведена к однозначно разрешимому уравнению Фредгольма второго рода в банаховом пространстве. Третья краевая задача и смешанная задача Дирихле-Неймана - это частные случаи рассмотренной задачи.

Библиография: 22 названия.
\end{abstract}

1. Введение. В настоящей работе продолжаются исследования, начатые в [1]-[3] и посвященные изучению смешанных краевых задач на плоскости. Рассматривается смешанная краевая задача для уравнения Лапласа во внешней многосвязной области. Все простые замкнутые кривые, составляющие границу, разбиты на три совокупности. На элементах первой из них задано условие Дирихле, на элементах второй - третье краевое условие, а третья, в свою очередь, разбита на две совокупности простых разомкнутых дуг, на элементах одной из которых задано условие Дирихле, а на элементах другой - третье краевое условие. Задача сведена к однозначно разрешимому интегральному уравнению Фредгольма второго рода в банаховом пространстве. Полученное интегральное уравнение может быть решено приближенно численными методами, развитьми в [4], [5]. Третья краевая задача и смешанная задача Дирихле-Неймана - частные случаи рассмотренной задачи.

Смешанная задача Дирихле-Неймана с произвольным разбиением границы для уравнения Лапласа исследовалась в [6], [7] с использованием теории аналитических функций. Однако в [6], [7] не предлагается метод сведения указанной задачи к однозначно разрешимьм интегральным уравнениям. Действительно, в [6] доказана формальная теорема существования с использованием комплексной функции Грина многосвязной области, практическое построение которой представляет собой отдельную трудоемкую задачу. Интегральные уравнения, полученные в [7], не являются однозначно разрешимыми. Метод анализа смешанной задачи, предложенньй в настоящей работе, основан

Работа выполнена при частичной поддержке Российского фонда фундаментальных исследований, грант № 99-01-01063. 
на теории интегральных операторов, развитой для решения двумерных краевых задач в областях, ограниченных замкнутыми и разомкнутыми кривьми [8]-[12]. Оказывается, что эти интегральные операторы можно эффективно применять в смешанных задачах с произвольным разбиением гранищы.

2. Постановка задачи и теорема единственности. На плоскости $x=\left(x_{1}, x_{2}\right)$ $\in \mathbb{R}^{2}$ рассмотрим внешнюю связную область $\mathscr{D}$ с гранищей $\partial \mathscr{D}$, состоящей из конечного числа простых замкнутых кривых класса $C^{2, \lambda}, \lambda \in(0,1]$, не имеющих общих точек. Предположим, что замкнутые кривые, составляющие $\partial \mathscr{D}$, разбиты на три множества $\Gamma$, $\gamma^{1}$ и $\gamma^{2}$ так, что $\Gamma \cup \gamma^{1} \cup \gamma^{2}=\partial \mathscr{D}$. При этом множество $\gamma^{1}$ состоит из $N_{2}$ замкнутых кривых $\gamma_{1}^{1}, \ldots, \gamma_{N_{2}}^{1}$. Далее, пусть каждая из замкнутых кривьх, составляющих $\Gamma$, поделена на четное число $(\geqslant 2)$ простых разомкнутьх дуг. Предположим, что все разомкнутые дуги поделены на два множества $\Gamma^{1}$ и $\Gamma^{2}$ таким образом, что каждая дуга из $\Gamma^{1}$ имеет общие конщы только с дугами из $\Gamma^{2}$ и

$$
\Gamma^{1} \cup \Gamma^{2}=\Gamma, \quad \Gamma^{k}=\bigcup_{n=1}^{N_{1}} \Gamma_{n}^{k}, \quad k=1,2 .
$$

Через $\Gamma_{n}^{k}$ обозначена разомкнутая дуга из множества $\Gamma^{k}$. Множества $\Gamma^{1}$ и $\Gamma^{2}$ имеют одинаковое число $N_{1}$ разомкнутых дуг.

Пусть каждая замкнутая кривая, составляющая $\partial \mathscr{D}$, параметризована и в качестве параметра выступает длина дуги $s$, отсчитываемая от некоторой фиксированной точки так, что область $\mathscr{D}$ остается справа при возрастании параметра $s$. На кривых, составляющих $\Gamma$, в качестве такой точки возьмем конец какой-либо разомкнутой дуги. При этом каждой замкнутой кривой из $\partial \mathscr{D}$ отвечает отрезок на оси $O s$. Предположим, что отрезки оси $O s$, отвечающие разньм замкнутьм кривьп, не имеют общих точек, в том числе и концов. В частности, для разомкнутых дуг $\Gamma^{1}$ положим

$$
\Gamma_{n}^{1}=\left\{x: x=x(s)=\left(x_{1}(s), x_{2}(s)\right), \quad s \in\left[a_{n}^{1}, b_{n}^{1}\right]\right\}, \quad n=1, \ldots, N_{1} .
$$

Для совокупностей отрезков оси $O s$, отвечающих $\Gamma^{1}, \Gamma^{2}, \Gamma, \gamma^{1}, \gamma^{2}$, сохраним эти обозначения, т.е. $\Gamma^{1}, \Gamma^{2}, \Gamma, \gamma^{1}, \gamma^{2}$ соответственно.

Пусть $\gamma^{k}$ - совокупность отрезков оси $O s$, отвечающих одноименной совокупности простых замкнутых кривьх, где $k=1$ либо $k=2$. Будем говорить, что функция $\mathscr{F}(s)$ параметра $s$ принадлежит классу $C^{0}\left(\gamma^{k}\right)$ (или $C^{0, \lambda}\left(\gamma^{k}\right)$ ), если она непрерьвна (или гёльдерова с показателем $\lambda$ ) на любом из отрезков $\gamma^{k}$ и на концах каждого такого отрезка ее значения совпадают, т.е. при полном обходе соответствующей замкнутой кривой функция $\mathscr{F}(s)$ возвращается к своему первоначальному значению. Аналогично, $\mathscr{F}(s) \in C^{1, \lambda}\left(\gamma^{k}\right)$, если $\mathscr{F}(s) \in C^{0}\left(\gamma^{k}\right)$ и на $\gamma^{k}$ существует производная $\mathscr{F}^{\prime}(s) \in C^{0, \lambda}\left(\gamma^{k}\right)$.

Вектор касательной к $\partial \mathscr{D}$ в точке $x(s)$, указывающий направление возрастания параметра $s$, обозначим $\tau_{x}=(\cos \alpha(s), \sin \alpha(s))$, где $\cos \alpha(s)=x_{1}^{\prime}(s)$ и $\sin \alpha(s)=x_{2}^{\prime}(s)$. Через $\boldsymbol{n}_{\boldsymbol{x}}=(\sin \alpha(s),-\cos \alpha(s))$ обозначим нормаль к $\partial \mathscr{D}$ в точке $x(s)$. Вектор $\boldsymbol{n}_{\boldsymbol{x}}$ совпадет с $\tau_{x}$, если $\boldsymbol{n}_{x}$ повернуть на угол $\pi / 2$ против часовой стрелки. Следовательно, $\boldsymbol{n}_{\boldsymbol{x}}-$ внутренняя нормаль по отношению к области $\mathscr{D}$.

Будем говорить, что функция $u(x)$ принадлежит классу гладкости $\mathbf{K}$, если вьполнены следующие условия:

1) $u(x) \in C^{0}(\overline{\mathscr{D}}) \cap C^{2}(\mathscr{D})$; 
2) $\nabla u(x) \in C^{0}(\overline{\mathscr{D}} \backslash X)$, где $X$-множество концов разомкнутых дуг, составляющих $\Gamma^{1}$ и $\Gamma^{2}$, т.е.

$$
X=\bigcup_{n=1}^{N_{1}}\left(x\left(a_{n}^{1}\right) \cup x\left(b_{n}^{1}\right)\right) ;
$$

3) в окрестности каждой точки $x(d) \in X$ для некоторых констант $c>0, \varepsilon>-1$ справедливо неравенство

$$
|\nabla u| \leqslant c|x-x(d)|^{\varepsilon}
$$

где $x \rightarrow x(d), x \in \mathscr{D}$ и $d=a_{n}^{1}$ либо $d=b_{n}^{1}\left(n=1, \ldots, N_{1}\right)$.

Сформулируем смешанную задачу $\mathbf{U}$ для уравнения Лапласа.

Задача $\mathbf{U}$. Найти функцию $u(x)$ из класса $\mathbf{K}$, удовлетворяющую уравнению Лапласа

$$
\Delta u(x)=0, \quad x \in \mathscr{D}, \quad \Delta=\frac{\partial^{2}}{\partial x_{1}^{2}}+\frac{\partial^{2}}{\partial x_{2}^{2}}
$$

граничным условиям

$$
\begin{aligned}
& \left.u(x)\right|_{x(s) \in \gamma^{1}}=f_{1}(s), \quad \frac{\partial u(x)}{\partial \boldsymbol{n}_{x}}+\left.\beta(s) u(x)\right|_{x(s) \in \gamma^{2}}=f_{2}(s), \\
& \left.u(x)\right|_{x(s) \in \Gamma^{1}}=f_{1}(s), \quad \frac{\partial u(x)}{\partial \boldsymbol{n}_{x}}+\left.\beta(s) u(x)\right|_{x(s) \in \Gamma^{2}}=f_{2}(s),
\end{aligned}
$$

и условиям на бесконечности

$$
|u(x)| \leqslant \text { const, } \quad|\nabla u(x)|=o\left(|x|^{-1}\right), \quad|x|=\sqrt{x_{1}^{2}+x_{2}^{2}} \rightarrow \infty
$$

Функции $\beta(s), f_{1}(s), f_{2}(s)$ считаются заданными. При этом $\beta(s) \in C^{0}\left(\Gamma^{2} \cup \gamma^{2}\right)$ и $\beta(s) \leqslant 0$ для всех $s \in \Gamma^{2} \cup \gamma^{2}$. Кроме того, дополнительно потребуем вьполнения следующих условий

$$
\operatorname{mes}\left(\Gamma^{1} \cup \gamma^{1}\right)+\|\beta(s)\|_{C^{0}\left(\Gamma^{2} \cup \gamma^{2}\right)}>0, \quad \operatorname{mes}\left(\partial \mathscr{D} \backslash \gamma^{1}\right)>0
$$

Если $\mathscr{L}$ - совокупность кривых, то через $\operatorname{mes}(\mathscr{L})$ обозначается их суммарная длина.

Условия (6) исключают из рассмотрения задачи Неймана и Дирихле, которые изучены, например, в [6], [13]-[16].

Все условия задачи $\mathbf{U}$ понимаются в классическом смысле. Смешанная задача Дирихле-Неймана с произвольным разбиением границы есть частньй случай задачи $\mathbf{U}$ при $\beta(s) \equiv 0$. Третья краевая задача также частный случаи задачи $\mathbf{U}$.

Справедлива 
Теорема 1. Задача $\mathbf{U}$ имеет не более одного решения.

Покажем, что однородная задача имеет только тривиальное решение. Пусть $u_{0}(x)-$ решение однородной задачи $\mathbf{U}$. Пользуясь свойствами гладкости функции $u_{0}(x)$, однородными граничными условиями (3), (4) и условиями на бесконечности, запишем энергетическое тождество для $u_{0}(x)$ :

$$
\left\|\nabla u_{0}\right\|_{L_{2}(\mathscr{D})}^{2}=-\int_{\partial \mathscr{D}} u_{0}(x(s)) \frac{\partial}{\partial \boldsymbol{n}_{x}} u_{0}(x(s)) d s=-\int_{\Gamma^{2} \cup \gamma^{2}}|\beta(s)|\left|u_{0}(x(s))\right|^{2} d s \leqslant 0,
$$

где учтено, что $\beta(s) \leqslant 0$ при $s \in \Gamma^{2} \cup \gamma^{2}$. Отсюда $\left\|\nabla u_{0}\right\|_{L_{2}(\mathscr{D})}^{2}=0$, а значит, $u_{0}(x) \equiv$ const в $\overline{\mathscr{D}}$. Если $\|\beta(s)\|_{C^{0}\left(\Gamma^{2} \cup \gamma^{2}\right)} \neq 0$, т.е. существует точка $s_{0}$ такая, что $\beta(s) \neq 0$, то, как следует из энергетического тождества, $u_{0}\left(x\left(s_{0}\right)\right)=0$. В этом случае const $=0$ и $u_{0}(x) \equiv 0$ в $\overline{\mathscr{D}}$. Если же $\beta(s) \equiv 0$, то согласно первому условию из (6) $\Gamma^{1} \cup \gamma^{1} \neq \varnothing$. В этом случае, пользуясь однородньм условием Дирихле из $(3),(4)$, получим const $=0$ и $u_{0}(x) \equiv 0$ в $\overline{\mathscr{D}}$.

Таким образом, однородная задача имеет только тривиальное решение. Из линейности задачи $\mathbf{U}$ вытекает, что неоднородная задача имеет не более одного решения. Тем самым, теорема доказана.

3. Сведение задачи к интегральным уравнениям. Пусть $\mathscr{L}_{m} \subset \partial \mathscr{D}_{\text {и }} \mathscr{B}_{m}\left(\mathscr{L}_{m}\right)$ - банахово пространство функций, определенных на множестве $\mathscr{L}_{m}$, где $m=1, \ldots, M$. Тогда для функций, определенных на $\bigcup_{m=1}^{M} \mathscr{L}_{m}$, рассмотрим банахово пространство $\bigcap_{m=1}^{M} \mathscr{B}_{m}\left(\mathscr{L}_{m}\right)$ с нормой

$$
\|\cdot\|_{\bigcap_{m=1}^{M} \mathscr{B}_{m}\left(\mathscr{L}_{m}\right)}=\sum_{m=1}^{M}\|\cdot\|_{\mathscr{B}_{m}\left(\mathscr{L}_{m}\right)} .
$$

При этом функции из $\bigcap_{m=1}^{M} \mathscr{B}_{m}\left(\mathscr{L}_{m}\right)$ принадлежат каждому из пространств $\mathscr{B}_{1}\left(\mathscr{L}_{1}\right)$, $\ldots, \mathscr{B}_{M}\left(\mathscr{L}_{M}\right)$.

Пусть в граничных условиях (3), (4) выполнены свойства

$$
\begin{gathered}
f_{1}(s) \in C^{1, \lambda}\left(\Gamma^{1}\right) \cap C^{1, \lambda}\left(\gamma^{1}\right), \\
\beta(s), f_{2}(s) \in C^{0, \lambda}\left(\Gamma^{2}\right) \cap C^{0, \lambda}\left(\gamma^{2}\right), \quad \lambda \in(0,1] .
\end{gathered}
$$

Здесь показатель Гёльдера $\lambda$ тот же, что и в определении гладкости границы $\partial \mathscr{D}$. Если на практике эти показатели различны, то в качестве $\lambda$ всегда можно взять наименьший.

Будем говорить, что $\mu(s) \in C_{q}^{\omega}\left(\Gamma^{1}\right) \mathrm{c} \omega \in(0,1], q \in[0,1)$, если

$$
\mu(s) \prod_{n=1}^{N_{1}}\left|s-a_{n}^{1}\right|^{q}\left|s-b_{n}^{1}\right|^{q} \in C^{0, \omega}\left(\Gamma^{1}\right),
$$

где $C^{0, \omega}\left(\Gamma^{1}\right)$ - пространство Гёльдера с показателем $\omega$ и

$$
\|\mu(s)\|_{C_{q}^{\omega}\left(\Gamma^{1}\right)}=\left\|\mu(s) \prod_{n=1}^{N_{1}}\left|s-a_{n}^{1}\right|^{q}\left|s-b_{n}^{1}\right|^{q}\right\|_{C^{0, \omega}\left(\Gamma^{1}\right)} .
$$


Положим

$$
\Phi(x, y)=-\frac{1}{2 \pi} \ln |x-y|, \quad|x-y|=\sqrt{\left(x_{1}-y_{1}\right)^{2}+\left(x_{2}-y_{2}\right)^{2}} .
$$

Ищем решение задачи $\mathbf{U}$ в следующем виде

$$
u[\mu](x)=w_{1}[\mu](x)+w_{2}[\mu](x)+v[\mu](x)+c[\mu]+h[\mu](x),
$$

где

$$
w_{1}[\mu](x)=\int_{\Gamma^{1}} \mu(\sigma) \Phi(x, y(\sigma)) d \sigma, \quad w_{2}[\mu](x)=\int_{\Gamma^{2} \cup \gamma^{2}} \mu(\sigma) \Phi(x, y(\sigma)) d \sigma
$$

- потенциалы простого слоя на $\Gamma^{1}$ и $\Gamma^{2} \cup \gamma^{2}$,

$$
v[\mu](x)=\int_{\gamma^{1}} \mu(\sigma) \frac{\partial \Phi(x, y(\sigma))}{\partial \boldsymbol{n}_{y}} d \sigma,
$$

- потенциал двойного слоя на $\gamma^{1}$,

$$
c[\mu]=\int_{\partial \mathscr{D}} \mu(s) d s
$$

- константа. Функцию $h[\mu](x)$ определим, основываясь на условиях (6). Второе из условий (6) говорит о том, что множество $\Gamma \cup \gamma^{2}$ не пусто и содержит по крайней мере одну замкнутую кривую. Внутри этой кривой выберем произвольную фиксированную точку $Y_{0}$. Пусть $Y_{1}, \ldots, Y_{N_{2}}$ - произвольные фиксированные точки, лежащие внутри кривых $\gamma_{1}^{1}, \ldots, \gamma_{N_{2}}^{1}$. Через $h[\mu](x)$ обозначим следующую сумму точечных источников, помешенньх в точки $Y_{0}, Y_{1}, \ldots, Y_{N_{2}}$ :

$$
h[\mu](x)=-\int_{\partial \mathscr{D}} \mu(s) d s \Phi\left(x, Y_{0}\right)+\sum_{n=1}^{N_{2}} \int_{\gamma_{n}^{1}} \mu(s) d s \Phi\left(x, Y_{n}\right) .
$$

Плотность $\mu(s)$ будем искать в следующем банаховом пространстве:

$$
\mu(s) \in C_{q}^{\omega}\left(\Gamma^{1}\right) \cap C^{0, \omega_{2}}\left(\Gamma^{2} \cup \gamma^{2}\right) \cap C^{1, \omega_{1}}\left(\gamma^{1}\right), \quad q \in[0,1), \quad \omega, \omega_{1}, \omega_{2} \in(0,1] .
$$

Как следует из свойств потенциала простого слоя [17], [18, § 2.5, 3.10], при такой плотности $\mu(s)$ потенциалы $w_{1}[\mu](x), w_{2}[\mu](x)$ принадлежат классу К. При этом если $q \in(0,1)$, то условия (1) выполнены с показателем $\varepsilon=-q$. Кроме того, $v[\mu](x)$ принадлежит $C^{1}(\overline{\mathscr{D}}) \cap C^{2}(\mathscr{D}) \subset \mathbf{K}$ как потенциал двойного слоя с плотностью, имеющей гёльдеровую производную $[18, \S 2.5,3.10]$. Этот результат можно получить иначе, а именно, представляя потенциал двойного слоя через угловой потенциал с помощью интегрирования по частям и затем пользуясь свойствами углового потенциала [17], [19]. Поскольку

$$
h[\mu](x) \in C^{\infty}\left(R^{2} \backslash\left(\bigcup_{n=0}^{N_{2}} Y_{n}\right)\right) \subset \mathbf{K},
$$

то $u[\mu](x) \in \mathbf{K}$. 
Очевидно, что $u[\mu](x)$ удовлетворяет уравнению $(2)$ в $\mathscr{D}$. Функция $h[\mu](x)$ построена таким образом, что $u[\mu](x)$ удовлетворяет условию на бесконечности (5). Тем самьм, функция $u[\mu](x)$ удовлетворяет всем условиям задачи $\mathbf{U}$, за исключением граничных условий.

Подставим потенциал $u[\mu](x)$ в граничные условия (3), (4) задачи $\mathbf{U}$ и получим граничные интегральные уравнения относительно плотности $\mu(s)$ на $\partial \mathscr{D}$ :

$$
\begin{aligned}
& \int_{\Gamma \cup \gamma^{2}} \mu(\sigma) \Phi(x(s), y(\sigma)) d \sigma+\frac{1}{2} \mu(s) \delta\left(s, \gamma^{1}\right) \\
& \quad+\int_{\gamma^{1}} \mu(\sigma) \frac{\partial \Phi(x(s), y(\sigma))}{\partial \boldsymbol{n}_{y}} d \sigma+c[\mu]+h[\mu](x(s))=f_{1}(s), \quad s \in \Gamma^{1} \cup \gamma^{1}, \\
& -\frac{1}{2} \mu(s)+\int_{\Gamma^{2} \cup \gamma^{2}} \mu(\sigma)\left(\frac{\partial}{\partial \boldsymbol{n}_{x}}+\beta(s)\right) \Phi(x(s), y(\sigma)) d \sigma \\
& \quad+\int_{\Gamma^{1}} \mu(\sigma)\left(\frac{\partial}{\partial \boldsymbol{n}_{x}}+\beta(s)\right) \Phi(x(s), y(\sigma)) d \sigma \\
& \quad+\int_{\gamma^{1}} \mu(\sigma)\left(\frac{\partial}{\partial \boldsymbol{n}_{x}}+\beta(s)\right) \frac{\partial}{\partial \boldsymbol{n}_{y}} \Phi(x(s), y(\sigma)) d \sigma \\
& \quad+\beta(s) c[\mu]+\left(\frac{\partial}{\partial \boldsymbol{n}_{x}}+\beta(s)\right) h[\mu](x(s))=f_{2}(s), \quad s \in \Gamma^{2} \cup \gamma^{2} .
\end{aligned}
$$

Здесь и далее под $\delta(s, \mathscr{L})$, где $\mathscr{L} \subset \partial \mathscr{D}$, понимается характеристическая функция множества $\mathscr{L}$, т.е.

$$
\delta(s, \mathscr{L})= \begin{cases}0, & \text { если } s \notin \mathscr{L} \\ 1, & \text { если } s \in \mathscr{L} .\end{cases}
$$

Пусть $\mu(s)$ - произвольное решение интегральных уравнений (9) из

$$
C_{q}^{\omega}\left(\Gamma^{1}\right) \cap C^{0}\left(\partial \mathscr{D} \backslash \Gamma^{1}\right), \quad q \in[0,1), \quad \omega \in(0,1] .
$$

Покажем, что оно принадлежит

$$
C_{q}^{\omega}\left(\Gamma^{1}\right) \cap C^{0, \omega_{2}}\left(\Gamma^{2} \cup \gamma^{2}\right) \cap C^{1, \omega_{1}}\left(\gamma^{1}\right), \quad q \in[0,1), \omega, \omega_{1}, \omega_{2} \in(0,1] .
$$

Рассмотрим уравнение (9b). Второй и третий интегралы в $(9 \mathrm{~b})$ принадлежат $C^{0, \lambda_{0}}\left(\Gamma^{2}\right.$ $\left.\cup \gamma^{2}\right)$ по $s$, где $\lambda_{0} \in(0, \min \{\lambda, 1-q\})$. Рассмотрим первый интегральный член в $(9 \mathrm{~b})$. Если $s \in \Gamma^{2} \cup \gamma^{2}$, то, как показано в [17], [20],

$$
\frac{\partial}{\partial \boldsymbol{n}_{x}} \Phi(x(s), y(\sigma)) \in C^{0, \lambda}\left(\left(\Gamma^{2} \cup \gamma^{2}\right) \times\left(\Gamma^{2} \cup \gamma^{2}\right)\right),
$$

потому что $\partial \mathscr{D} \in C^{2, \lambda}$. Кроме того,

$$
\int_{\Gamma^{2} \cup \gamma^{2}} \mu(\sigma) \Phi(x(s), y(\sigma)) d \sigma \in C^{0,1 / 2-\varepsilon_{0}}\left(\Gamma^{2} \cup \gamma^{2}\right)
$$

при любом $\varepsilon_{0} \in(0,1 / 2)$. Действительно,

$$
\int_{\Gamma^{2} \cup \gamma^{2}} \mu(\sigma) \Phi(x(s), y(\sigma)) d \sigma=-\frac{1}{2 \pi} \int_{\Gamma^{2} \cup \gamma^{2}} \mu(\sigma) \frac{\mathscr{I}_{1}(x(s), y(\sigma))}{|x(s)-y(\sigma)|^{1 / 2+\varepsilon_{0}}} d \sigma,
$$


где $\varepsilon_{0}-$ произвольное число из интервала $(0,1 / 2)$, и с учетом $[13, \S 6.2]$

$$
\mathscr{I}_{1}(x(s), y(\sigma))=|x(s)-y(\sigma)|^{1 / 2+\varepsilon_{0}} \ln |x(s)-y(\sigma)| \in C^{0,1 / 2}\left(\left(\Gamma^{2} \cup \gamma^{2}\right) \times\left(\Gamma^{2} \cup \gamma^{2}\right)\right) .
$$

В силу $[13, \S 51]$ функция в правой части (11) принадлежит $C^{0,1 / 2-\varepsilon_{0}}\left(\Gamma^{2} \cup \gamma^{2}\right)$ по $s$, если $\mu(s) \in C^{0}\left(\gamma^{2} \cup \Gamma^{2}\right)$. Следовательно, утверждение, содержащееся в (10), доказано. Поскольку $\varepsilon_{0}$ можно взять сколь угодно малым, то первый интегральньй член в $(9 \mathrm{~b})$ принадлежит $C^{0, \omega_{2}}\left(\Gamma^{2} \cup \gamma^{2}\right)$ при любом $\omega_{2}$ из интервала

$$
(0, \min \{\lambda, 1 / 2,1-q\})
$$

если плотность $\mu(s) \in C^{0}\left(\Gamma^{2} \cup \gamma^{2}\right)$.

Тем самьм, если $\mu(s) \in C_{q}^{\omega}\left(\Gamma^{1}\right) \cap C^{0}\left(\partial \mathscr{D} \backslash \Gamma^{1}\right)$, то суммарный интегральньй член в $(9 \mathrm{~b})$ принадлежит $C^{0, \omega_{2}}\left(\Gamma^{2} \cup \gamma^{2}\right)$ с $\omega_{2}$ из интервала (12). Если, кроме того, $\mu(s)-$ решение уравнения (9b), обращающее его в тождество, то из этого тождества с учетом условий (7) вытекает, что $\mu(s) \in C_{q}^{\omega}\left(\Gamma^{1}\right) \cap C^{0, \omega_{2}}\left(\Gamma^{2} \cup \gamma^{2}\right) \cap C^{0}\left(\gamma^{1}\right)$.

Покажем, что всякое интегрируемое на $\partial \mathscr{D} \backslash \gamma^{1}$ и непрерьвное на $\gamma^{1}$ решение $\mu(s)$ интегрального уравнения (9a) принадлежит $C^{1, \lambda / 2}\left(\gamma^{1}\right)$. Действительно, если $s \in \gamma^{1}$, то суммарньй интегральный член в уравнении (9a) имеет производную, так как $\gamma^{1} \in C^{2, \lambda}$. Эта производная представляется в виде несобственного интеграла с ядром вида

$$
\frac{\mathscr{I}_{2}(s, \sigma)}{|s-\sigma|^{1-\lambda / 2}}+\mathscr{I}_{3}(s, \sigma), \quad \mathscr{I}_{3}(s, \sigma), \mathscr{I}_{2}(s, \sigma) \in C^{0, \lambda / 2}\left(\gamma^{1} \times \partial \mathscr{D}\right) .
$$

В силу $[13, \S 51]$ указанная производная принадлежит $C^{0, \lambda / 2}\left(\gamma^{1}\right)$ по $s$. Поскольку $f_{1}(s) \in$ $C^{1, \lambda}\left(\gamma^{1}\right)$ в соответствии с $(7)$, то решение $\mu(s)$ уравнения (9a) принадлежит $C^{1, \lambda / 2}\left(\gamma^{1}\right)$.

Таким образом, всякое решение $\mu(s)$ интегральных уравнений (9) в классе

$$
C_{q}^{\omega}\left(\Gamma^{1}\right) \cap C^{0}\left(\partial \mathscr{D} \backslash \Gamma^{1}\right)
$$

автоматически принадлежит $C_{q}^{\omega}\left(\Gamma^{1}\right) \cap C^{0, \omega_{2}}\left(\Gamma^{2} \cup \gamma^{2}\right) \cap C^{1, \lambda / 2}\left(\gamma^{1}\right)$ с $\omega_{2}$ из интервала (12). При этом, как следует из приведенных рассуждений, $u[\mu](x)$ удовлетворяет всем условиям задачи U. Таким образом, доказана

ТЕОРема 2. Пусть выполнены условия (7). Если интегральные уравнения (9) имеют решение $\mu(s)$ в $C_{q}^{\omega}\left(\Gamma^{1}\right) \cap C^{0}\left(\partial \mathscr{D} \backslash \Gamma^{1}\right)$ c $\omega \in(0,1], q \in[0,1)$, то

1) $\mu(s) \in C_{q}^{\omega}\left(\Gamma^{1}\right) \cap C^{0, \omega_{2}}\left(\Gamma^{2} \cup \gamma^{2}\right) \cap C^{1, \lambda / 2}\left(\gamma^{1}\right)$ при любом $\omega_{2}$ из интервала (12),

2) решение задачи $\mathbf{U}$ существует и дается формулой (8).

Задача $\mathbf{U}$ сведена к интегральным уравнениям (9). На $\Gamma^{1}$ это уравнение первого рода с логарифмическим ядром, а на $\Gamma^{2}$ это уравнение второго рода. Ниже исследуем разрешимость интегральных уравнений $(9)$ в классе $C_{q}^{\omega}\left(\Gamma^{1}\right) \cap C^{0}\left(\partial \mathscr{D} \backslash \Gamma^{1}\right)$, для чего сведем их к уравнению Фредгольма второго рода.

Дифференцируем интегральное уравнение (9a) по $s$ на $\Gamma^{1}$, тогда на $\Gamma^{1}$ получим сингулярное интегральное уравнение

$$
\begin{aligned}
\frac{\partial}{\partial s} u[\mu](x(s))= & \frac{1}{2 \pi} \int_{\Gamma^{1}} \mu(\sigma) \frac{\sin \varphi_{0}(x(s), y(\sigma))}{|x(s)-y(\sigma)|} d \sigma+\int_{\Gamma^{2} \cup \gamma^{2}} \mu(\sigma) \frac{\partial}{\partial s} \Phi(x(s), y(\sigma)) d \sigma \\
& +\int_{\gamma^{1}} \mu(\sigma) \frac{\partial}{\partial s} \frac{\partial}{\partial n_{y}} \Phi(x(s), y(\sigma)) d \sigma+\frac{\partial}{\partial s} h[\mu](x(s))=f_{1}^{\prime}(s), \quad s \in \Gamma^{1},
\end{aligned}
$$


где $\varphi_{0}(x, y)$ - угол между векторами $\vec{x} y$ и $\boldsymbol{n}_{x}$. Угол $\varphi_{0}(x, y)$ считается положительным, если он отсчитывается от $\boldsymbol{n}_{x}$ против часовой стрелки, и отрицательным, если он отсчитьвается от $\boldsymbol{n}_{x}$ по часовой стрелке. Первьй интеграл в (13a) понимается в смысле главного значения по Коши.

Уравнения (9) на $\Gamma^{2} \cup \gamma^{1} \cup \gamma^{2}$ запишем в виде

$$
\mu(s)+\int_{\partial \mathscr{D}} \mu(\sigma) A_{2}(s, \sigma) d \sigma=2 \delta\left(s, \gamma^{1}\right) f_{1}(s)-2 \delta\left(s, \Gamma^{2} \cup \gamma^{2}\right) f_{2}(s), \quad s \in \partial \mathscr{D} \backslash \Gamma^{1},
$$

где

$$
\begin{aligned}
A_{2}(s, \sigma)=2 & \delta\left(s, \gamma^{1}\right)\left[\delta\left(\sigma, \gamma^{1}\right) \frac{\partial}{\partial \boldsymbol{n}_{y}} \Phi(x(s), y(\sigma))+\delta\left(\sigma, \Gamma \cup \gamma^{2}\right) \Phi(x(s), y(\sigma))\right. \\
& \left.+1-\Phi\left(x(s), Y_{0}\right)+\sum_{n=1}^{N_{2}} \delta\left(\sigma, \gamma_{n}^{1}\right) \Phi\left(x(s), Y_{n}\right)\right] \\
- & 2 \delta\left(s, \Gamma^{2} \cup \gamma^{2}\right)\left[\delta\left(\sigma, \Gamma \cup \gamma^{2}\right)\left(\frac{\partial}{\partial \boldsymbol{n}_{x}}+\beta(s)\right) \Phi(x(s), y(\sigma))\right. \\
& +\delta\left(\sigma, \gamma^{1}\right)\left(\frac{\partial}{\partial \boldsymbol{n}_{x}}+\beta(s)\right) \frac{\partial}{\partial \boldsymbol{n}_{y}} \Phi(x(s), y(\sigma)) \\
& +\beta(s)-\left(\frac{\partial}{\partial \boldsymbol{n}_{x}}+\beta(s)\right) \Phi\left(x(s), Y_{0}\right) \\
& \left.+\sum_{n=1}^{N_{2}} \delta\left(\sigma, \gamma_{n}^{1}\right)\left(\frac{\partial}{\partial \boldsymbol{n}_{x}}+\beta(s)\right) \Phi\left(x(s), Y_{n}\right)\right] .
\end{aligned}
$$

Функция $A_{2}(s, \sigma)$ непрерывна, если $s \in \gamma^{1}, \sigma \in \partial \mathscr{D}$, а при $s \in \Gamma^{2} \cup \gamma^{2}, \sigma \in \partial \mathscr{D}$ представима в виде

$A_{2}(s, \sigma)=\frac{1}{\pi} \beta(s) \delta\left(\sigma, \Gamma \cup \gamma^{2}\right) \ln |(x(s)-y(\sigma))|+\mathscr{I}_{0}(s, \sigma), \quad \mathscr{I}_{0}(s, \sigma) \in C^{0}\left(\left(\Gamma^{2} \cup \gamma^{2}\right) \times \partial \mathscr{D}\right)$,

т.е. ядро $A_{2}(s, \sigma)$ может иметь логарифмическую особенность, если $s=\sigma \in \Gamma^{2} \cup \gamma^{2}$. Тем самьм, интегральный оператор в $(13 \mathrm{~b})$ с ядром $A_{2}(s, \sigma)$ действует из $C_{q}^{\omega}\left(\Gamma^{1}\right) \cap$ $C^{0}\left(\partial \mathscr{D} \backslash \Gamma^{1}\right)$ в $C^{0}\left(\partial \mathscr{D} \backslash \Gamma^{1}\right)$. Очевидно, $A_{2}(s, \sigma) \in C^{0}\left(\left(\partial \mathscr{D} \backslash \Gamma^{1}\right) \times \gamma^{1}\right)$, апри $\sigma \in \Gamma \cup \gamma^{2}$, $s \in \partial \mathscr{D} \backslash \Gamma^{1}$ ядро $A_{2}(s, \sigma)$ можно записать в виде

$$
A_{2}(s, \sigma)=\mathscr{I}_{4}(s, \sigma)|x(s)-y(\sigma)|^{-\varepsilon_{1}},
$$

где $\mathscr{I}_{4}(s, \sigma)$ принадлежит $C^{0}\left(\left(\partial \mathscr{D} \backslash \Gamma^{1}\right) \times\left(\Gamma \cup \gamma^{2}\right)\right)$ при любом $\varepsilon_{1} \in(0,1)$.

Уравнение (13a) эквивалентно исходному интегральному уравнению (9a) на $\Gamma^{1}$ тогда и только тогда, когда (13a) дополнено следующими условиями

$$
u[\mu]\left(x\left(a_{n}^{1}\right)\right)=f_{1}\left(a_{n}^{1}\right), \quad n=1, \ldots, N_{1} .
$$

Система (13) эквивалентна исходным интегральным уравнениям (9).

Легко показать [17], что

$$
\frac{\sin \varphi_{0}(x(s), y(\sigma))}{|x(s)-y(\sigma)|}-\frac{1}{\sigma-s} \in C^{0, \lambda}\left(\Gamma^{1} \times \Gamma^{1}\right) .
$$


Следовательно, перепишем (13a) в виде

$$
2 \frac{\partial}{\partial s} u[\mu](x(s))=\frac{1}{\pi} \int_{\Gamma^{1}} \frac{\mu(\sigma)}{\sigma-s} d \sigma+\int_{\partial \mathscr{D}} \mu(\sigma) M(s, \sigma) d \sigma=2 f_{1}^{\prime}(s), \quad s \in \Gamma^{1},
$$

где

$$
\begin{aligned}
& M(s, \sigma)=\delta\left(\sigma, \Gamma^{1}\right) \frac{1}{\pi}\left(\frac{\sin \varphi_{0}(x(s), y(\sigma))}{|x(s)-y(\sigma)|}-\frac{1}{\sigma-s}\right)+2 \delta\left(\sigma, \Gamma^{2} \cup \gamma^{2}\right) \frac{\partial}{\partial s} \Phi(x(s), y(\sigma)) \\
& \quad+2 \delta\left(\sigma, \gamma^{1}\right) \frac{\partial}{\partial s} \frac{\partial}{\partial n_{y}} \Phi(x(s), y(\sigma))-2 \frac{\partial}{\partial s} \Phi\left(x(s), Y_{0}\right)+2 \sum_{n=1}^{N_{2}} \delta\left(\sigma, \gamma_{n}^{1}\right) \frac{\partial}{\partial s} \Phi\left(x(s), Y_{n}\right)
\end{aligned}
$$

Таким образом, система уравнений (9) сведена к эквивалентной системе (13), разрешимость которой будет доказана в следующем пункте.

4. Интегральное уравнение Фредгольма второго рода и теорема существования. Обращая сингулярный интегральньй оператор в (14), получим следующее интегральное уравнение второго рода [13]:

$$
\mu(s)+\frac{1}{Q_{1}(s)} \int_{\partial \mathscr{D}} \mu(\sigma) A_{1}(s, \sigma) d \sigma+\frac{1}{Q_{1}(s)} \sum_{n=0}^{N_{1}-1} G_{n} s^{n}=\frac{1}{Q_{1}(s)} F_{1}(s), \quad s \in \Gamma^{1},
$$

где

$$
\begin{gathered}
A_{1}(s, \sigma)=-\frac{1}{\pi} \int_{\Gamma^{1}} \frac{M(\xi, \sigma)}{\xi-s} Q_{1}(\xi) d \xi, \quad Q_{1}(s)=\prod_{n=1}^{N_{1}}\left|\sqrt{s-a_{n}^{1}} \sqrt{b_{n}^{1}-s}\right| \operatorname{sign}\left(s-a_{n}^{1}\right), \\
F_{1}(s)=-\frac{1}{\pi} \int_{\Gamma^{1}} \frac{2 Q_{1}(\sigma) f_{1}^{\prime}(\sigma)}{\sigma-s} d \sigma
\end{gathered}
$$

и $G_{0}, \ldots, G_{N_{1}-1}-$ произвольные постоянные.

Используя свойства сингулярных интегралов [17], [13], можно показать, что всякое решение уравнения (15) принадлежит $C_{1 / 2}^{\omega}\left(\Gamma^{1}\right)$, поэтому ниже мы ищем $\mu(s)$ на $\Gamma^{1}$ в этом пространстве.

Положим

$$
Q(s)=\delta\left(s, \Gamma^{1}\right) Q_{1}(s)+\delta\left(s, \partial \mathscr{D} \backslash \Gamma^{1}\right), \quad s \in \partial \mathscr{D} .
$$

Вместо $\mu(s) \in C_{1 / 2}^{\omega}\left(\Gamma^{1}\right) \cap C^{0}\left(\partial \mathscr{D} \backslash \Gamma^{1}\right)$ введем новую неизвестную функцию

$$
\mu_{*}(s)=\mu(s) Q(s) \in C^{0, \omega}\left(\Gamma^{1}\right) \cap C^{0}\left(\partial \mathscr{D} \backslash \Gamma^{1}\right)
$$

и запишем $(15),(13 \mathrm{~b})$ в форме одного уравнения второго рода на всей границе $\partial \mathscr{D}$ :

$$
\mu_{*}(s)+\int_{\partial \mathscr{D}} \mu_{*}(\sigma) Q^{-1}(\sigma) A(s, \sigma) d \sigma+\delta\left(s, \Gamma^{1}\right) \sum_{n=0}^{N_{1}-1} G_{n} s^{n}=F(s), \quad s \in \partial \mathscr{D}
$$

где

$$
\begin{aligned}
A(s, \sigma) & =\delta\left(s, \Gamma^{1}\right) A_{1}(s, \sigma)+\delta\left(s, \partial \mathscr{D} \backslash \Gamma^{1}\right) A_{2}(s, \sigma) \\
F(s) & =\delta\left(s, \Gamma^{1}\right) F_{1}(s)+2 \delta\left(s, \gamma^{1}\right) f_{1}(s)-2 \delta\left(s, \Gamma^{2} \cup \gamma^{2}\right) f_{2}(s) .
\end{aligned}
$$


Выберем константы $G_{0}, \ldots, G_{N_{1}-1}$ так, чтобы удовлетворить условиям $(13 \mathrm{c})$. Для этого подставим $\mu(s)$ из $(13 \mathrm{~b}),(15)$ в $(13 \mathrm{c})$, тогда в терминах $\mu_{*}(s)$ получим

$$
\int_{\partial \mathscr{D}} Q^{-1}(\xi) \mu_{*}(\xi) l_{n}(\xi) d \xi+\sum_{m=0}^{N_{1}-1} B_{n m} G_{m}=H_{n}, \quad n=1, \ldots, N_{1}
$$

где

$$
\begin{aligned}
l_{n}(\xi) & =-u\left[Q^{-1}(\cdot) A(\cdot, \xi)\right]\left(a_{n}^{1}\right) \\
B_{n m} & =-u\left[Q^{-1}(\cdot) \delta\left(\cdot, \Gamma^{1}\right)(\cdot)^{m}\right]\left(a_{n}^{1}\right) \\
H_{n} & =-u\left[Q^{-1}(\cdot) F(\cdot)\right]\left(a_{n}^{1}\right)+f_{1}\left(a_{n}^{1}\right) .
\end{aligned}
$$

Точкой · обозначается переменная интегрирования в потенциале (8).

Таким образом, система уравнений (13) для $\mu(s)$ сведена к системе $(16)$ относительно функции $\mu_{*}(s)$ и констант $G_{0}, \ldots, G_{N_{1}-1}$. Очевидно, всякое решение системы (16) дает решение системы (13).

Пользуясь схемой рассуждений, приведенной в [17], можно показать, что $F_{1}(s) \in$ $C^{0, p}\left(\Gamma^{1}\right), p=\min \{1 / 2, \lambda\}$ и интегральньй оператор в $(16 \mathrm{a})$ действует из $C^{0}(\partial \mathscr{D})$ в $C^{0, p}\left(\Gamma^{1}\right) \cap C^{0}\left(\partial \mathscr{D} \backslash \Gamma^{1}\right)$. Тем самым, доказана

Лемма 1. Пусть $F(s) \in C^{0, p}\left(\Gamma^{1}\right) \cap C^{0}\left(\partial \mathscr{D} \backslash \Gamma^{1}\right), p=\min \{\lambda, 1 / 2\}$. Ecлu $\mu_{*}(s) \in$ $C^{0}(\partial \mathscr{D})$ удовлетворяет уравнению $(16 \mathrm{a})$, то функиия $\mu_{*}(s)$ принадлежит классу $C^{0, p}\left(\Gamma^{1}\right) \cap C^{0}\left(\partial \mathscr{D} \backslash \Gamma^{1}\right)$.

Условие $F(s) \in C^{0, p}\left(\Gamma^{1}\right) \cap C^{0}\left(\partial \mathscr{D} \backslash \Gamma^{1}\right)$ вьполнено, если выполнены условия (7). Следовательно, ниже будем искать $\mu_{*}(s)$ в $C^{0}(\partial \mathscr{D})$.

В силу специального представления для функции $A_{2}(s, \sigma)$, приведенного после $(13 \mathrm{~b})$, интегральньй оператор с ядром $A_{2}(s, \sigma) Q^{-1}(\sigma)$ компактен как оператор, действующий из $C^{0}(\partial \mathscr{D})$ в $C^{0}\left(\partial \mathscr{D} \backslash \Gamma^{1}\right)$. Действительно, компактность из $C^{0}\left(\gamma^{1} \cup \Gamma\right)$ в $C^{0}\left(\partial \mathscr{D} \backslash \Gamma^{1}\right)$ следует из теоремы Арцела, а компактность из $C^{0}\left(\gamma^{2}\right)$ в $C^{0}\left(\partial \mathscr{D} \backslash \Gamma^{1}\right)$ вытекает из компактности операторов с полярньм ядром [21, с. 170, теорема 7.4.1].

Так же как и в $[17]$, можно показать, что интегральньй оператор в (16a)

$$
\boldsymbol{A} \mu_{*}=\int_{\partial \mathscr{D}} \mu_{*}(\sigma) Q^{-1}(\sigma) A(s, \sigma) d \sigma
$$

компактно отображает $C^{0}(\partial \mathscr{D})$ в себя.

Перепишем (16a) в операторной форме

$$
(I+\boldsymbol{A}) \mu_{*}+P G=F,
$$

где $P$ - оператор умножения строки $P=\delta\left(s, \Gamma^{1}\right)\left(s^{0}, \ldots, s^{N_{1}-1}\right)$ на столбец $G=\left(G_{0}, \ldots\right.$ $\left.\ldots, G_{N_{1}-1}\right)^{T}$. Оператор $P$ конечномерньй из $E_{N_{1}}$ в $C^{0}(\partial \mathscr{D})$ и потому компактньй [22].

Теперь запишем уравнения $(16 \mathrm{~b})$ в форме

$$
I_{N_{1}} G+L \mu_{*}+\left(B-I_{N_{1}}\right) G=H
$$


где $H=\left(H_{1}, \ldots, H_{N_{1}}\right)^{T}$ - столбец из $N_{1}$ элементов, $I_{N_{1}}$ - тождественньй оператор в $E_{N_{1}}, B$ - матрища размерности $\left(N_{1} \times N_{1}\right)$, состоящая из элементов $B_{n m}$ из (17). Оператор $L$ действует из $C^{0}(\partial \mathscr{D})$ в $E_{N_{1}}$, так что $L \mu_{*}=\left(L_{1} \mu_{*}, \ldots, L_{N_{1}} \mu_{*}\right)^{T}$, где

$$
L_{n} \mu_{*}=\int_{\partial \mathscr{D}} Q^{-1}(\xi) \mu_{*}(\xi) l_{n}(\xi) d \xi, \quad n=1, \ldots, N_{1}
$$

Операторы $\left(B-I_{N_{1}}\right), L$ - конечномерные, а потому компактные [22].

Рассмотрим столбцы

$$
\tilde{\mu}=\left(\begin{array}{c}
\mu_{*} \\
G
\end{array}\right), \quad \widetilde{F}=\left(\begin{array}{c}
F \\
H
\end{array}\right)
$$

в банаховом пространстве $C^{0}(\partial \mathscr{D}) \times E_{N_{1}}$ с нормой

$$
\|\tilde{\mu}\|_{C^{0}(\partial \mathscr{D}) \times E_{N_{1}}}=\left\|\mu_{*}\right\|_{C^{0}(\partial \mathscr{D})}+\|G\|_{E_{N_{1}}} .
$$

Запишем систему (18) в форме одного уравнения

$$
(\boldsymbol{I}+\boldsymbol{R}) \tilde{\mu}=\widetilde{F}, \quad \boldsymbol{R}=\left(\begin{array}{cc}
\boldsymbol{A} & P \\
L & B-I_{N_{1}}
\end{array}\right),
$$

где $\boldsymbol{I}$ - тождественный оператор в пространстве $C^{0}(\partial \mathscr{D}) \times E_{N_{1}}$. Очевидно, $\boldsymbol{R}$ - компактный оператор, отображающий $C^{0}(\partial \mathscr{D}) \times E_{N_{1}}$ в себя. Следовательно, $(19)$ - уравнение фредгольма в этом пространстве.

Покажем, что однородное уравнение (19) имеет только тривиальное решение. Тогда в силу альтернативы Фредгольма неоднородное уравнение (19) имеет единственное решение при любой правой части. Доказательство проведем методом от противного. Пусть

$$
\tilde{\mu}^{0}=\left(\begin{array}{c}
\mu_{*}^{0} \\
G^{0}
\end{array}\right) \in C^{0}(\partial \mathscr{D}) \times E_{N_{1}}
$$

- нетривиальное решение однородного уравнения (19). По лемме 1

$$
\tilde{\mu}^{0}=\left(\begin{array}{c}
\mu_{*}^{0} \\
G^{0}
\end{array}\right) \in C^{0, p}\left(\Gamma^{1}\right) \cap C^{0}\left(\partial \mathscr{D} \backslash \Gamma^{1}\right) \times E_{N_{1}}, \quad p=\min \{\lambda, 1 / 2\} .
$$

Следовательно, функция $\mu^{0}(s)=\mu_{*}^{0}(s) Q^{-1}(s) \in C_{1 / 2}^{p}\left(\Gamma^{1}\right) \cap C^{0}\left(\partial \mathscr{D} \backslash \Gamma^{1}\right)$ и столбец $G^{0}$ преврашают однородные уравнения $(13 \mathrm{~b}),(15),(16 \mathrm{~b})$ в тождества. Например, (13b) принимает вид

$$
\lim _{\substack{x \rightarrow x(s) \in \gamma^{1} \\ x \in \mathscr{D}}} u\left[\mu^{0}\right](x)=0, \quad \lim _{\substack{x \rightarrow x(s) \in \Gamma^{2} \cup \gamma^{2} \\ x \in \mathscr{D}}}\left(\frac{\partial}{\partial \boldsymbol{n}_{x}}+\beta(s)\right) u\left[\mu^{0}\right](x)=0,
$$

где потенциал $u\left[\mu^{0}\right](x)$ определяется формулой (8). Используя однородные тождества $(13 \mathrm{~b}),(15)$, проверим, что однородные тож дества (16b) эквивалентны следующим

$$
u\left[\mu^{0}\right]\left(a_{n}^{1}\right)=0, \quad n=1, \ldots, N_{1} .
$$


Кроме того, действуя на однородное тождество (15) сингулярным интегральным оператором с ядром $(s-t)^{-1}$, найдем, что $\mu^{0}(s)$ удовлетворяет однородному уравнению (13a), т.е.

$$
\left.\frac{\partial}{\partial s} u\left[\mu^{0}\right](x(s))\right|_{\Gamma^{1}}=0 .
$$

Из $(20)$ вытекает, что $\mu^{0}(s)$ удовлетворяет однородным уравнениям (9). По теореме 2 $u\left[\mu^{0}\right](x)$ - решение однородной задачи $\mathbf{U}$ и $\mu^{0}(s) \in C_{1 / 2}^{p}\left(\Gamma^{1}\right) \cap C^{0, \omega_{2}}\left(\Gamma^{2} \cup \gamma^{2}\right) \cap C^{1, \lambda / 2}\left(\gamma^{1}\right)$ при любом $\omega_{2}$ из интервала (12). По теореме 1

$$
u\left[\mu^{0}\right](x) \equiv 0, \quad x \in \overline{\mathscr{D}}
$$

Заметим, что $\lim _{|x| \rightarrow \infty} u\left[\mu^{0}\right](x)=c\left[\mu^{0}\right]=0$, т.е.

$$
\int_{\partial \mathscr{D}} \mu^{0}(s) d s=0
$$

Следовательно, точечный источник в точке $Y_{0}$ отсутствует и

$$
h\left[\mu^{0}\right](x)=\sum_{n=1}^{N_{2}} \int_{\gamma_{n}^{1}} \mu^{0}(s) d s \Phi\left(x, Y_{n}\right)
$$

так что внутри замкнутых кривых, составляющих $\Gamma \cup \gamma^{2}$, точечных источников нет.

Пусть $\mathscr{L}$ - произвольная замкнутая кривая, принадлежащая $\Gamma \cup \gamma^{2}$ и ограничивающая внутреннюю односвязную область $\Omega$. Функция $u\left[\mu^{0}\right](x)$ в окрестности $\mathscr{L}$ представляется потенциалом простого слоя с гладкими добавками и поэтому непрерьвна (не имеет скачка) при переходе через $\mathscr{L}$. Очевидно, что в $\bar{\Omega}$ функция $u\left[\mu^{0}\right](x)$ удовлетворяет однородной задаче Дирихле:

$$
\Delta u(x)=0, \quad x \in \Omega,\left.\quad u(x(s))\right|_{\mathscr{L}}=0 .
$$

Принимая во внимание гладкость функции $\mu^{0}(s)$ и свойства потенциала простого слоя $\left[17\right.$, теорема 4], убеждаемся в том, что $u\left[\mu^{0}\right](x) \in C^{0}(\bar{\Omega}) \cap C^{2}(\Omega)$. Согласно принципу максимума для гармонических функций [15] однородная задача Дирихле (24) имеет только тривиальное решение: $u\left[\mu^{0}\right](x) \equiv 0$ в $\bar{\Omega}$. Пользуясь $(21)$ и теоремой о скачке для нормальной производной потенциала простого слоя на $\mathscr{L}$, получим

$$
\lim _{\substack{x \rightarrow x(s) \in \mathscr{L} \\ x \in \mathscr{D}}} \frac{\partial u\left[\mu^{0}\right](x)}{\partial \boldsymbol{n}_{x}}-\lim _{\substack{x \rightarrow x(s) \in \mathscr{L} \\ x \in \Omega}} \frac{\partial u\left[\mu^{0}\right](x)}{\partial \boldsymbol{n}_{x}}=-\mu^{0}(s) \equiv 0,
$$

т.е. $\mu^{0}(s) \equiv 0$ на $\mathscr{L}$. Так как $\mathscr{L}$-произвольная замкнутая кривая, составляюшая $Г \cup \gamma^{2}$, то $\mu^{0}(s) \equiv 0$ на $\Gamma \cup \gamma^{2}$. Тем самым, $u\left[\mu^{0}\right](x)$ принимает вид

$$
u\left[\mu^{0}\right](x)=\int_{\gamma^{1}} \mu^{0}(\sigma) \frac{\partial \Phi(x, y(\sigma))}{\partial \boldsymbol{n}_{y}} d \sigma+\sum_{n=1}^{N_{2}} \int_{\gamma_{n}^{1}} \mu^{0}(\sigma) d \sigma \Phi\left(x, Y_{n}\right)
$$


и в соответствии с $(22)$

$$
\int_{\gamma^{1}} \mu^{0}(s) d s=0 .
$$

Пусть $\mathscr{D}_{n}-$ внутренняя односвязная область, ограниченная кривой $\gamma_{n}^{1}$, и $\mathscr{D}_{0}=\bigcup_{n=1}^{N_{2}} \mathscr{D}_{n}$. Из приведенных рассуждений вытекает, что

$$
u\left[\mu^{0}\right](x) \equiv 0, \quad x \in \mathbb{R}^{2} \backslash\left(\mathscr{D}_{0} \cup \gamma^{1}\right) .
$$

Рассмотрим произвольную простую гладкую замкнутую кривую $\mathscr{J}$, лежащую в области $\mathbb{R}^{2} \backslash\left(\mathscr{D}_{0} \cup \gamma^{1}\right)$ и охватьвающую из всех кривых, составляюших $\gamma^{1}$, только кривую $\gamma_{n}^{1}$ $\left(n=1, \ldots, N_{2}\right)$. Полагая, что $\boldsymbol{n}_{x}$ - непрерьвное поле нормалей на кривой $\mathscr{J}$, выгислим

$$
\begin{aligned}
\int_{\mathscr{J}} \frac{\partial u\left[\mu^{0}\right](x)}{\partial \boldsymbol{n}_{\boldsymbol{x}}} d l_{x}=0= & \int_{\gamma_{n}^{1}} \mu^{0}(\sigma) \frac{\partial}{\partial \boldsymbol{n}_{y}}\left(\int_{\mathscr{J}} \frac{\partial \Phi(x, y(\sigma))}{\partial \boldsymbol{n}_{x}} d l_{x}\right) d \sigma \\
& +\int_{\gamma_{n}^{1}} \mu^{0}(\sigma) d \sigma \int_{\mathscr{J}} \frac{\partial \Phi\left(x, Y_{n}\right)}{\partial \boldsymbol{n}_{x}} d l_{x}
\end{aligned}
$$

Здесь учтено следующее свойство гармонических функций: если функция $\mathscr{U}(x)$ гармоническая внутри контура $\mathscr{J}$ и $\nabla \mathscr{U}(x)$ непрерывно продолжается на $\mathscr{J}$ изнутри, то

$$
\int_{\mathscr{J}} \frac{\partial \mathscr{U}}{\partial n_{x}} d l_{x}=0
$$

Примем во внимание, что

$$
\left.\int_{\mathscr{J}} \frac{\partial \Phi(x, y(\sigma))}{\partial \boldsymbol{n}_{x}}\right|_{y(\sigma) \in \gamma_{n}^{1}} d l_{x}=\int_{\mathscr{J}} \frac{\partial \Phi\left(x, Y_{n}\right)}{\partial \boldsymbol{n}_{x}} d l_{x}= \pm 1
$$

где знак зависит от выбора направления поля нормалей $\boldsymbol{n}_{\boldsymbol{x}}$ на кривой $\mathscr{J}$. Отсюда вытекает равенство

$$
\int_{\mathscr{J}} \frac{\partial u\left[\mu^{0}\right](x)}{\partial \boldsymbol{n}_{x}} d l_{x}= \pm \int_{\gamma_{n}^{1}} \mu^{0}(\sigma) d \sigma=0 .
$$

Поскольку наши рассуждения справедливы для любого $n=1, \ldots, N_{2}$, то убеждаемся в справедливости следующих тождеств:

$$
\int_{\gamma_{n}^{1}} \mu^{0}(\sigma) d \sigma=0, \quad n=1, \ldots, N_{2} .
$$

Функция $u\left[\mu^{0}\right](x)$ принимает вид потенциала двойного слоя:

$$
u\left[\mu^{0}\right](x)=\int_{\gamma^{1}} \mu^{0}(\sigma) \frac{\partial \Phi(x, y(\sigma))}{\partial \boldsymbol{n}_{y}} d \sigma .
$$

По теореме 2 функция $\mu^{0}(\sigma) \in C^{1, \lambda / 2}\left(\gamma^{1}\right)$. Как показано в [18], при такой плотности $\mu^{0}(\sigma)$ первые производные потенциала двойного слоя $u\left[\mu^{0}\right](x)$ непрерывно продолжимы на $\gamma_{n}^{1}\left(n=1, \ldots, N_{2}\right)$ извне и изнутри. Более того,

$$
\lim _{\substack{x \rightarrow x(s) \in \gamma_{n}^{1} \\ x \in \mathscr{D}}} \frac{\partial u\left[\mu^{0}\right](x)}{\partial \boldsymbol{n}_{x}}=\lim _{\substack{x \rightarrow x(s) \in \gamma_{n}^{1} \\ x \in \mathscr{D}_{n}}} \frac{\partial u\left[\mu^{0}\right](x)}{\partial \boldsymbol{n}_{x}}=0, \quad n=1, \ldots, N_{2},
$$


где учтено (25). Очевидно, функция $u\left[\mu^{0}\right](x)$ удовлетворяет следующей задаче Неймана в $\mathscr{D}_{n}$ :

$$
\Delta u=0, \quad x \in \mathscr{D}_{n},\left.\quad \frac{\partial u}{\partial \boldsymbol{n}_{x}}\right|_{\gamma_{n}^{1}}=0, \quad n=1, \ldots, N_{2} .
$$

Известно [15], что решением такой задачи является константа, т.е.

$$
u\left[\mu^{0}\right](x) \equiv c_{n}, \quad x \in \mathscr{D}_{n}, \quad n=1, \ldots, N_{2},
$$

где $c_{1}, \ldots, c_{N_{2}}$ - некоторые константы. Пользуясь теоремой о скачке потенциала двойного слоя [15] и пользуясь (25), получим

$$
\lim _{\substack{x \rightarrow x(s) \in \gamma_{n}^{1} \\ x \in \mathscr{D}}} u\left[\mu^{0}\right](x)-\lim _{\substack{x \rightarrow x(s) \in \gamma_{n}^{1} \\ x \in \mathscr{D}_{n}}} u\left[\mu^{0}\right](x)=\mu^{0}(s)=-c_{n}, \quad n=1, \ldots, N_{2},
$$

так что $\mu^{0}(s) \equiv-c_{n}$ на $\gamma_{n}^{1}\left(n=1, \ldots, N_{2}\right)$. Из $(26)$ вытекает, что $c_{n}=0$ при любом $n=1, \ldots, N_{2}$. Следовательно, $\mu^{0}(s) \equiv 0$ на $\gamma^{1}$.

Таким образом, $\mu^{0}(s) \equiv 0$ на $\partial \mathscr{D}$ и $\mu_{*}^{0}(s)=Q(s) \mu^{0}(s) \equiv 0$ на $\partial \mathscr{D}$. Из однородного тождества $(16 \mathrm{a})$ для функции $\mu_{*}^{0}(s)$ и констант $G_{0}^{0}, \ldots, G_{N_{1}-1}^{0}$ получим

$$
G^{0}=\left(G_{0}^{0}, \ldots, G_{N_{1}-1}^{0}\right)^{T} \equiv 0 .
$$

Тем самым,

$$
\tilde{\mu}^{0}=\left(\begin{array}{c}
\mu_{*}^{0} \\
G^{0}
\end{array}\right) \equiv 0
$$

и мы приходим к противоречию с предположением о том, что $\tilde{\mu}^{0}$ - нетривиальное решение однородного уравнения (19). В результате однородное уравнение (19) имеет только тривиальное решение. По теоремам Фредгольма неоднородное уравнение (19) однозначно разрешимо в $C^{0}(\partial \mathscr{D}) \times E_{N_{1}}$. Итак, нами доказана

ТЕОРема 3. Уравнение (19) (или система (16)) в пространстве $C^{0}(\partial \mathscr{D}) \times E_{N_{1}}$ есть уравнение Фредгольма второго рода, которое при любой правой части

$$
\widetilde{F}=\left(\begin{array}{c}
F \\
H
\end{array}\right) \in C^{0}(\partial \mathscr{D}) \times E_{N_{1}}
$$

имеет единственное решение

$$
\tilde{\mu}=\left(\begin{array}{c}
\mu_{*} \\
G
\end{array}\right) \in C^{0}(\partial \mathscr{D}) \times E_{N_{1}}
$$

Напомним, что если выполнены условия (7), то $F(s) \in C^{0, p}\left(\Gamma^{1}\right) \cap C^{0}\left(\partial \mathscr{D} \backslash \Gamma^{1}\right)$, $p=\min \{\lambda, 1 / 2\}$, и по лемме 1 решение $\tilde{\mu}$ уравнения (21), гарантированное теоремой 3 , принадлежит $C^{0, p}\left(\Gamma^{1}\right) \cap C^{0}\left(\partial \mathscr{D} \backslash \Gamma^{1}\right) \times E_{N_{1}}$.

Как отмечено вьше, если функция $\mu_{*}(s) \in C^{0, p}\left(\Gamma^{1}\right) \cap C^{0}\left(\partial \mathscr{D} \backslash \Gamma^{1}\right)$ и константы $G_{0}, \ldots$ $\ldots, G_{N_{1}-1}$ удовлетворяют системе $(16)$, то функция $\mu(s)=Q^{-1}(s) \mu_{*}(s) \in C_{1 / 2}^{p}\left(\Gamma^{1}\right) \cap$ $C^{0}\left(\partial \mathscr{D} \backslash \Gamma^{1}\right)$ - решение системы $(13)$ и, следовательно, $\mu(s)$ удовлетворяет уравнениям (9). Из приведенных рассуждений вытекает 
ТЕОРема 4. Если выполнены условия (7), то уравнения (9) имеют решение $\mu(s)$ в $C_{1 / 2}^{p}\left(\Gamma^{1}\right) \cap C^{0}\left(\partial \mathscr{D} \backslash \Gamma^{1}\right)$ с $p=\min \{\lambda, 1 / 2\}$. При этом справедливо представление $\mu(s)=\mu_{*}(s) Q^{-1}(s)$, где $\mu_{*}(s) \in C^{0, p}\left(\Gamma^{1}\right) \cap C^{0}\left(\partial \mathscr{D} \backslash \Gamma^{1}\right) ;$ оно находится $\boldsymbol{\theta}$ результате решения уравнения Фредгольма второго рода (19) (или системы (16)), которое однозначно разрешимо.

Напомним, что функция $Q(s)$ введена в уравнении (16a).

ЗАмЕчаниЕ. Так же, как и при доказательстве теоремы 3, можно показать, что решение интегральных уравнений (9) (или эквивалентной системы (13)), гарантированное теоремой 4 , единственно в $C_{1 / 2}^{p_{1}}\left(\Gamma^{1}\right) \cap C^{0}\left(\partial \mathscr{D} \backslash \Gamma^{1}\right)$ при любом $p_{1} \in(0, p]$. Это замечание может быть полезно при проведении численных расчетов, так как решение интегральных уравнений может быть получено дискретизацией и прямым численным обращением матрицы [4], [5].

На основе теорем 2,4 приходим к окончательному результату.

ТЕОРема 5. Если выполнены условия (7), то решение задачи $\mathbf{U}$ существует и дается формулой $(8)$, где $\mu(s)$ - решение уравнений $(9)$ в $C_{1 / 2}^{p}\left(\Gamma^{1}\right) \cap C^{0}\left(\partial \mathscr{D} \backslash \Gamma^{1}\right)$, $p=\min \{\lambda, 1 / 2\}$, гарантированное теоремой 4 . Более точно, $\mu(s) \in C_{1 / 2}^{p}\left(\Gamma^{1}\right) \cap$ $C^{0, \omega_{2}}\left(\Gamma^{2} \cup \gamma^{2}\right) \cap C^{1, \lambda / 2}\left(\gamma^{1}\right)$ при любом $\omega_{2}$ из интервала (12).

Отметим, что согласно свойствам потенциалов из [17] решение задачи $\mathbf{U}$ удовлетворяет оценке (1) с $\varepsilon=-q=-1 / 2$. Явные формулы, описьвающие поведение градиента решения в окрестности конщов разомкнутых дуг, составляющих $\Gamma$, несложно получить, воспользовавшись результатами работ [17], [20].

Теорема 5 гарантирует существование классического решения задачи $\mathbf{U}$, когда $\partial \mathscr{D} \in$ $C^{2, \lambda}$ и функции $\beta(s), f_{1}(s), f_{2}(s)$ из граничных условий $(3),(4)$ удовлетворяют условиям (7). Единственность классического решения вытекает из теоремы 1 . На основании полученных результатов можно предложить следующую схему решения задачи $\mathbf{U}$. Сначала находим единственное решение уравнения Фредгольма (19) (или системы (16)) в $C^{0}(\partial \mathscr{D}) \times E_{N_{1}}$. Это решение автоматически принадлежит

$$
C^{0, p}\left(\Gamma^{1}\right) \cap C^{0}\left(\partial \mathscr{D} \backslash \Gamma^{1}\right) \times E_{N_{1}}, \quad p=\min \left\{\lambda, \frac{1}{2}\right\}
$$

Далее, строим решение уравнений $(9)$ в $C_{1 / 2}^{p}\left(\Gamma^{1}\right) \cap C^{0}\left(\partial \mathscr{D} \backslash \Gamma^{1}\right)$ с помощью соотношения $\mu(s)=\mu_{*}(s) Q^{-1}(s)$. Это решение автоматически принадлежит

$$
C_{1 / 2}^{p}\left(\Gamma^{1}\right) \cap C^{0, \omega_{2}}\left(\Gamma^{2} \cup \gamma^{2}\right) \cap C^{1, \lambda / 2}\left(\gamma^{1}\right)
$$

при любом $\omega_{2}$ из интервала (12). Окончательно, подставляя $\mu(s)$ в $(8)$, получаем решение задачи $\mathbf{U}$.

В соответствии с замечанием к теореме 4 единственное решение уравнений (9) (или эквивалентной системы $(13))$ в $C_{1 / 2}^{p_{1}}\left(\Gamma^{1}\right) \cap C^{0}\left(\partial \mathscr{D} \backslash \Gamma^{1}\right)$ с $p_{1} \in(0, p]$ может быть найдено напрямую, непосредственным решением указанньх уравнений. 


\section{СПИСОК ЦИТИРОВАННОЙ ЛИТЕРАТУРЫ}

[1] Крутицкий П. А. О смешанной задаче для уравнения Гельмгольца в многосвязной области // ЖВМиМФ. 1996. Т. 36. № 8. С. 127-137.

[2] Крутицкий П. А. Смешанная задача для уравнения Гельмгольца вне разрезов на плоскости // Дифференц. уравнения. 1996. Т. 32. №9. С. 1153-1162.

[3] Крутицкий П. А. Смешанная задача для уравнения Лапласа вне разрезов на плоскости // Дифференц. уравнения. 1997. Т. 33. № 9. С. 1181-1190.

[4] Белоцерковский С. М., Лифанов И. К. Численные методы в сингулярных интегральных уравнениях. М.: Наука, 1985.

[5] Лифанов И. К. Метод сингулярных интегральных уравнений и численный эксперимент. М.: Янус, 1995.

[6] Гринберг В. М., Цлаф Л.Я. (ред. ) Интегральные уравнения. М., 1968.

[7] Homentcovschi D. On the Dirichlet-Neumann mixed boundary value problem for harmonic functions in plane multiply connected domains // Rev. Romaine Math. Pures Appl. 1987. V. 32. № 9. P. 801-810.

[8] Krutitskii P. A. Fast stratified flow over several obstacles, including wings // IMA J. Appl. Math. 1996. V. 57. №3. P. 243-256.

[9] Krutitskii P.A. The 2-D Neumann problem in an exterior domain bounded by closed and open curves // Math. Methods Appl. Sci. 1997. V. 20. № 18. P. 1551-1562.

[10] Krutitskii P. A. Wave propagation in a 2-D external domain bounded by closed and open curves // Nonlinear Analysis, Theory, Methods and Applications. 1998. V. 32. № 1. P. 135-144.

[11] Krutitskii P. A. The Neumann problem for the 2-D Helmholtz equation in a domain, bounded by closed and open curves // Internat. J. Math. Math. Sci. 1998. V. 21. № 2. P. 209-216.

[12] Krutitskii P. A. The Dirichlet problem for the dissipative Helmholtz equation in a plane domain bounded by closed and open curves // Hiroshima Math. J. 1998. V. 28. № 1. P. 149-168.

[13] Мусхелишвили Н.И. Сингулярные интегральные уравнения. М.: Наука, 1968.

[14] Jacob C. Introduction mathematique a la mecanique des fluides. Bucarest-Paris, 1959.

[15] Владимиров В. С. Уравнения математической физики. М.: Наука, 1981.

[16] Крутицкий П. А. Вторая начально-краевая задача для уравнения гравитационно-гироскопических волн во внешней области // Матем. заметки. 1996. Т. 60. №1. С. 40-57.

[17] Крутицкий П. А. Задача Дирихле для уравнения Гельмгольца вне разрезов на плоскости // ЖВМиМФ. 1994. Т. 34. № 8-9. С. 1237-1258.

[18] Колтон Д., Кресс Р. Методы интегральных уравнений в теории рассеяния. М.: Мир, 1987.

[19] Габов С.А. Угловой потенциал и его некоторые приложения // Матем. сб. 1977. T. 103 (145). № 4. C. 490-504.

[20] Крутицкий П. А. Задача Неймана для уравнения Гельмгольца вне разрезов на плоскости // ЖВМиМФ. 1994. Т. 34. № 11. С. 1652-1665.

[21] Михлин С. Г. Курс математической физики. М.: Наука, 1968.

[22] Крейн С. Г. (ред.) Функциональный анализ. М.: Наука, 1964.

Московский государственный университет им. М.В.Ломоносова

Поступило

ИПМ им. Келдыша РАН

30.08.1998

Исправленный вариант

23.08.1999 